\title{
Efficient Linearly Polarized Lamp for Liquid Crystal Displays
}

\author{
Chang-Ching Tsai and Shin-Tson Wu, Fellow, IEEE
}

\begin{abstract}
We propose a new optical design to produce linearly polarized illumination for liquid crystal displays. The operation mechanism is based on polarization conversion due to induced surface plasmon polariton on metallic gratings in conical diffraction. This linearly polarized lamp integrates an unpolarized light source and a light recycling scheme inside the lamp housing. Its structure is simple and, moreover, its optical efficiency reaches $92 \%$ in emitting a linearly polarized light.
\end{abstract}

Index Terms-Backlight recycling, linear polarized light source, polarization conversion via surface plasmons.

\section{INTRODUCTION}

$\mathbf{L}$ INEARLY polarized light is commonly used in liquid crystal displays (LCDs) in order to achieve high contrast ratio [1]. To obtain linear polarization from an unpolarized backlight for direct-view displays or from an arc lamp for projection displays, a linear polarizer or polarizing beam splitter (PBS) is widely used. However, more than $50 \%$ of the incident light is lost by the employed polarizers. To recover the light loss, several light recycling schemes have been developed [2]-[5]. Basically, the recycling process includes a PBS (either cube or film) to transmit one polarization, say $p$-wave, from the unpolarized light source while reflecting $s$-wave back for recycling, and a polarization converter to convert the $s$-wave back to $p$-wave. All these schemes concern about the recycling of the light outside the illuminating source. As a result, several optical elements for light-guiding and polarization-converting are required and the light recycling system is bulky.

In this paper, we propose a simple and efficient polarization conversion scheme using induced surface plasmon polariton (SPP) on metallic gratings, in which light recycling takes place inside the lamp housing. Thus, the device is compact, lightweight, and potentially low cost. First, let us briefly discuss the polarization conversion through metallic gratings. It has been reported [6] that a linearly polarized light can rotate its polarization axis to a certain degree if the tangential incident wavevector on the metallic grating plane is neither parallel nor normal to the grating vector. Such polarization conversion is

Manuscript received November 20, 2008. Current version published January 09, 2009. This work was supported by Chi-Mei Optoelectronics Corporation (Taiwan).

The authors are with the College of Optics and Photonics, University of Central Florida, Orlando, FL 32816 USA (e-mail: tsaicc@ @ creol.ucf.edu; swu@ mail. ucf.edu).

Color versions of one or more of the figures in this paper are available online at http://ieeexplore.iee.org.

Digital Object Identifier 10.1109/JDT.2008.2010513

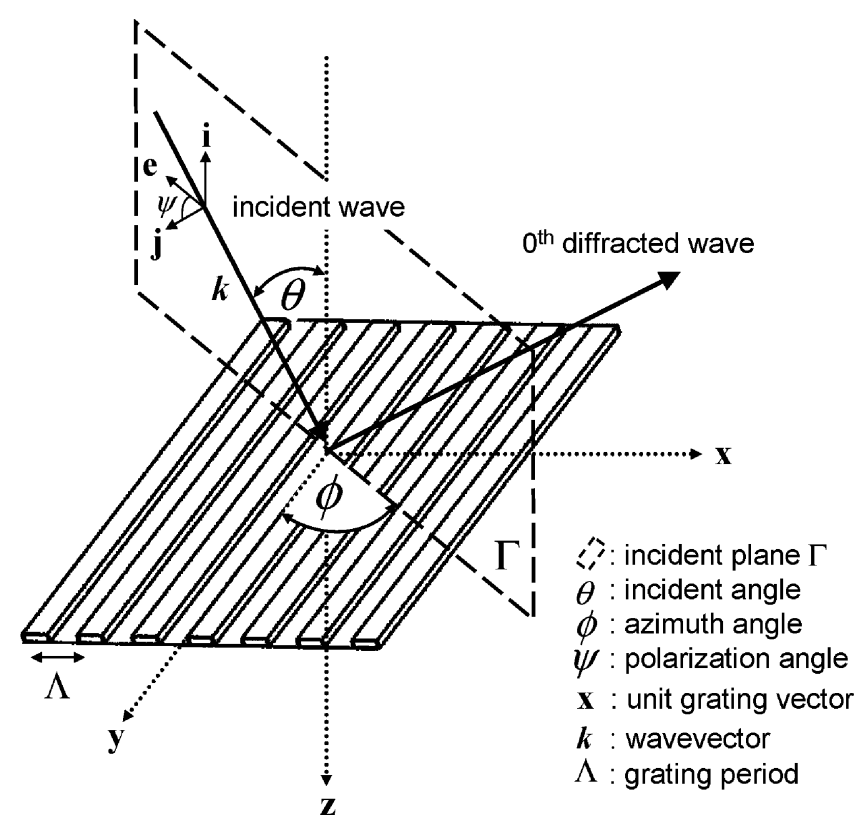

Fig. 1. Polarization conversion occurs in the conical diffraction when a linearly polarized light is reflected from the metallic grating with incident angle $\theta$, azimuthal angle $\phi$, polarization angle $\psi$, and grating period $\Lambda$.

due to the resonance of SPP with the diffracted light [7]. In particular, certain deep metallic gratings can cause both very high polarization conversion efficiency and broadband performance [8], [9]. Recently, an application of this SPP-type polarization converter for LCD backlight with a very high recycling efficiency was reported [10]. Typically, a highly reflective metal would excite stronger SPP due to the contribution of dense free electrons. Therefore, in practical applications the material used is usually silver- or aluminum-coated gratings. Since the reflector inside the lamp is also coated with metals, we then seek for an alternate possibility to integrate the polarization converter with the lamp structure inside the lamp housing.

\section{Device Configuration}

Fig. 1 indicates the necessary conical diffraction between the incident light and metallic gratings to induce SPP polarization conversion, where $\theta$ is the polar (incident) angle, $\phi$ is the azimuthal angle. The unit polarization vector is indicated by $\mathbf{e}$ and $\psi$ is the polarization angle (the angle between $\mathbf{e}$ and $\mathbf{j}$ in the incident plane $\Gamma$, where the unit normal vector $\mathbf{i}$ and tangential vector $\mathbf{j}$ with respect to $\Gamma$, satisfying $\mathbf{i} \times \mathbf{j}=\boldsymbol{k}$ ). With $\psi=0^{\circ}$ or $90^{\circ}$ it corresponds to $\boldsymbol{E}$ or $\boldsymbol{H}$ field that is perpendicular to the plane of incidence respectively. The grating is in the $\mathbf{x}-\mathbf{y}$ plane with grating vector along $\mathbf{x}$ and the incident plane $\Gamma$ is 

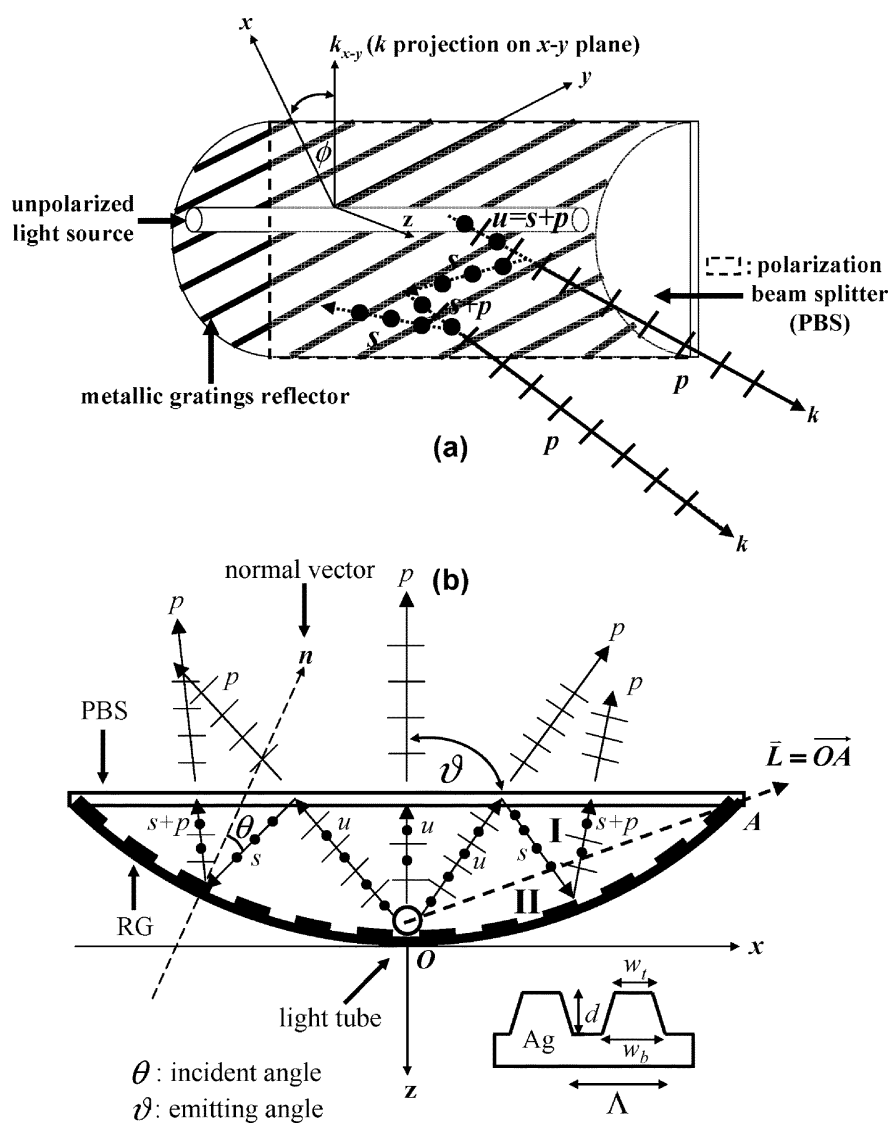

Fig. 2. (a) The front view of our polarizing lamp: a long light tube, a broadband wide-angle polarization beam splitter (PBS), and an arc-metallic grating reflector (GR). The initial unpolarized quasi-cylindrical $u$-wave passes the $p$-wave through PBS and reflects back $s$-wave to GR, where $s$-wave is rotated into elliptical $s+p$-wave. The total emitting power of $p$-wave is greatly enhanced by this light recycling process. (b) The side-view of the polarizing lamp. In simulation, the lamp dimension is determined by the parabolic reflective grating (RG), which is characterized by $z=a x^{2}$, with $a=0.04,0 \leq x \leq 5$, and $x \approx 10^{4} \lambda$. Inside the lamp, $\vec{L}=\overrightarrow{O A}$ divides the ray tracing area into zone $\mathbf{I}$ and II, respectively. The profile of the trapezoid grating is with the top $w_{t}=60 \mathrm{~nm}$, bottom $w_{b}=70 \mathrm{~nm}$, depth $d=124 \mathrm{~nm}$, and pitch $\Lambda=197 \mathrm{~nm}$, oriented with azimuthal angle $\phi=\pi / 4$.

determined by $\mathbf{k}$ and $\mathbf{z}$ vectors. For polarization conversion to occur, the azimuthal angle should satisfy $\phi \neq 0^{\circ}$ or $\phi \neq 90^{\circ}$.

Fig. 2(a) shows the front view of our polarizing lamp which consists of a long light tube as light source, a PBS film as lamp cover, and an arc-metallic grating as reflector, and Fig. 2(b) depicts the side view. The working principle is described as follows. First, the light tube emits a quasi-cylindrical $u$-wave in all directions. If the reflective grating is, for example, parabolic and the position of cross-section of the light tube is located at near the focus of the reflective grating, then the rays are supposed to pass through the PBS almost parallel. Initially, the PBS will transmit one polarization, say TM or $p$-wave, of the unpolarized light and rebound the other polarization, TE or $s$-wave, back to the reflective grating. Next, the light will undergo the recycling process. When the returned $s$-wave hits the reflective gratings, its polarization is changed to elliptical and reflected back toward PBS again. The ellipticity is characterized by its two elliptic axes with the respective TE and TM components. If a perfect polarization conversion occurs, namely a complete rotation of $s$-wave into $p$-wave, then the ellipse will be reduced to a line along the TM axis. We can say that high ellipticity represents a high polarization conversion value and gives better recycling efficiency. The PBS film will transmit the $p$-wave of the elliptically polarized light and bounce back the remaining $s$-wave. This is called one cycle of the light recycling. By repeating many such cycles, our polarizing lamp can emit a $p$-wave dominated light.

\section{RESULTS}

The groove shape and azimuthal orientation of reflective gratings are essential in determining the polarization conversion efficiency. The detailed mechanisms have been discussed in our previous publications [10], [11]. If the polarization conversion value is high, in one or two cycles, the lamp can output a fairly pure linearly polarized light. For simplicity, we consider the grating pitch $\Lambda$ in the subwavelength range of visible light, i.e. $\Lambda<\lambda$ so only the zeroth diffracted order is the propagation mode, while the others are evanescent waves. Besides, since the curvature radius $r$ of our arc reflector is much larger than the pitch, $\Lambda \ll r$, the grating can be treated as plane for each incident ray. Then the propagation of rays inside the lamp will follow the Snell's law. In such condition and ignoring the absorption from PBS (it is typically very small [12]), the total converted $p$-wave can be calculated as follows:

$$
\begin{aligned}
p_{0} & =1-s_{0}, p_{i \neq 0}=C_{s p, i} \times s_{i}, \\
s_{i+1} & =\left(R_{s, i}-C_{s p, i}\right) \times s_{i}, p_{\text {total }}=\sum_{i=0}^{\infty} p_{i}
\end{aligned}
$$

where $C_{s p, i}\left(\vartheta_{j}, \lambda_{k}\right)$ is the polarization conversion efficiency of $s$-wave converted to $p$-wave, $R_{s, i}\left(\vartheta_{j}, \lambda_{k}\right)$ is the reflectivity of grating for the incident $s$-ray of $\left(\vartheta_{j}, \lambda_{k}\right)$ in the $i$-th cycling process, $\vartheta_{j}$ is the emitting angle of each $j$-th cylindrical ray from the light tube in the range $0 \leq \vartheta_{j} \leq 360^{\circ}$, and $\lambda_{k}$ is the $k$-th wavelength in visible spectrum, $400 \leq \lambda_{k} \leq 700(\mathrm{~nm})$. Here the definition of $C_{s p, i}$ is the power carried by the reflected $p_{i}$-wave divided by that of the incident $s_{i}$-wave passing the propagating cross section. Typically, for a normalized randomly polarized light, we set the initial intensity $p_{0}=s_{0}=0.5$. In the simulation we present here, we choose the location of the light tube along the symmetric $z$-axis at the bottom of the silver reflective gratings as shown in Fig. 2(b). In this configuration, we only need to discuss $\vartheta_{j}$ in the range of $0 \leq \vartheta_{j} \leq 87^{\circ}$ due to symmetry with $z$-axis (for $\vartheta_{j}>87^{\circ}$ the ray cannot propagate out of lamp housing). There is a zone line $\vec{L}=\overrightarrow{O A}$ from the tube point $O$ to the lamp edge point $A$, which divides the lamp into two zones. In zone $\mathbf{I}$, the initial unpolarized light to use (1) is $p_{0}=s_{0}=0.5$, but in zone II the rays will hit the reflective gratings first before going into the PBS, so the initial unpolarized intensity for each $j, k$ ray should be calculated as following. We note that the reflectances of $p$-wave and $s$-wave are generally different, i.e. $R_{p}\left(\vartheta_{j}, \lambda_{k}\right) \neq R_{s}\left(\vartheta_{j}, \lambda_{k}\right)$. For $p$-wave, $R_{p}$ indeed is equal to the sum of the two components: $C_{p p}$ and $C_{p s}$, i.e., $R_{p}=C_{p p}+C_{p s}$, where $C_{p s}$ is the polarization converted and $C_{p p}$ is the efficiency of the polarization not converted. In 

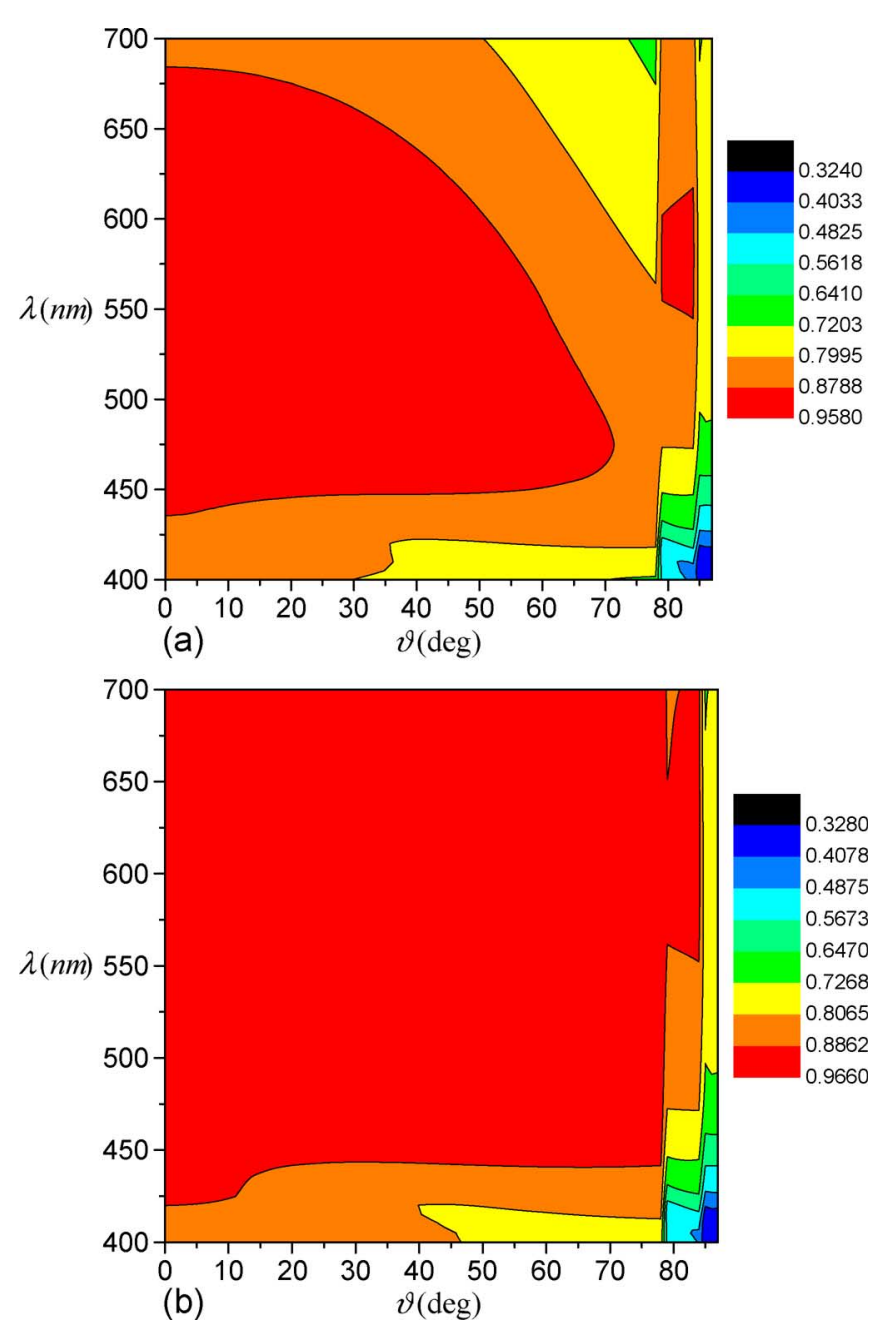

Fig. 3. (a) The total normalized emitting power of $p$-wave after one time light recycling for $400 \leq \lambda \leq 700(\mathrm{~nm})$ and $0 \leq \vartheta \leq 87^{\circ}$ with maximum $p_{1, \max }=95.63 \%$, minimum $p_{1, \min }=32.46 \%$ and average $\bar{p}_{1}=86.75 \%$. (b) The result of five times recycling, maximum $p_{5, \max }=96.49 \%$, minimum $p_{5, \text { min }}=32.92 \%$ and average $\bar{p}_{5}=92.17 \%$.

the same way, for $s$-wave $R_{s}=C_{s s}+C_{s p}$. Therefore, in zone II the initial unpolarized light in (1) is $p_{0}=0.5 \times\left(C_{p p}+C_{s p}\right)$ and $s_{0}=0.5 \times\left(C_{s s}+C_{p s}\right)$. It is proven that for the zeroth order propagating wave, the conversion efficiencies of $p \rightarrow s$ and $s \rightarrow p$ are equal, i.e. $C_{p s}=C_{s p}$ for symmetric gratings [13], [14], but $C_{s s} \neq C_{p p}$. Next, we show the numerical results of the total polarized power of the configuration in Fig. 2, where the shape of the groove is identical to the trapezoid grating used in [10]. The numerical method used here to calculate the various diffraction coefficients is the rigorous coupled wave analysis [11], [15], [16]. Inside the lamp, we follow the typical ray tracing technique to determine the light propagation. The dispersion and absorption of visible light with silver [17] are included in the calculation.

Fig. 3(a) and (b) give the total intensity of the outgoing $p$-wave in one and five cycles of light recycling, respectively. We see that most of the polarized power in $\left(\vartheta_{j}, \lambda_{k}\right)$ region can reach $80 \%$ both in one cycle and five cycles. For polarizing performance of this lamp, we define the figure of merit as an integrated average of the $p$-polarized power over the ranges of incident angle $\Delta \vartheta$ and wavelength $\Delta \lambda$ after $n$ cycles

$$
\bar{p}_{n}=\iint \sum_{i=0}^{n} p_{i}(\lambda, \vartheta) d \lambda d \vartheta / \Delta \lambda \Delta \vartheta .
$$

The average power for one and five cycles in Fig. 3 are $\bar{p}_{1}=86.75 \%$ and $\bar{p}_{5}=92.17 \%$, respectively. We now compare $\bar{p}_{5}$ with the emitting efficiency of the lamp $\bar{p}_{e}$, which is obtained by replacing the grating reflector with the same smooth arc-reflector and removing the PBS. On the other hand, $p_{e}(\lambda, \vartheta)$ represents the unpolarized illuminating power from the lamp. Similar to (2), we can calculate $\bar{p}_{e}=\int p_{e} d \vartheta d \lambda / \Delta \vartheta \Delta \lambda$. Now with the same incident condition, the calculated result is $\bar{p}_{e}=98.58 \%$, which means we can transform an unpolarized lamp into a linearly polarized light source with high efficiency $\left(\bar{p}_{5} / \bar{p}_{e}=93.50 \%\right)$. This figure demonstrates that our lamp is a very high energy-saving device to provide linearly polarized illumination. This linearly polarized lamp is particularly useful for projection displays using LCD panels [2].

\section{CONCLUSION}

We have utilized the surface plasmon polariton-aided polarization conversion on metallic gratings to design a new lamp, which can emit a linearly polarized light with rather high efficiency. Comparing with other polarization conversion schemes, this lamp has several advantages. 1) It does not need any light guide, which is generally made of several prisms and reflectors. Therefore, it greatly reduces the complexity of the display system. 2) The power efficiency is almost unbeatable. Since inside a lamp there always needs a reflector embedded, which causes certain inevitable power loss, now we use the same reflector with grooving not only to reflect the light but also get the polarization converted. In this way, we do not need additional polarization conversion elements such that the loss is controlled to minimum. The multi-function of grating reflector is the key element for saving energy. 3) The whole light recycling process is integrated inside the lamp house, which will make this device compact and lightweight. This feature is particularly attractive for portable devices, e.g., micro- projectors, cellular phones, and other mobile displays.

\section{REFERENCES}

[1] D. K. Yang and S. T. Wu, Fundamentals of Liquid Crystal Devices. Hoboken, NJ: Wiley, 2006.

[2] D. Armitage, I. Underwood, and S. T. Wu, Introduction to Microdisplays. Hoboken, NJ: Wiley, 2006.

[3] W. C. Yip, H. C. Huang, and H. S. Kwok, "Efficient polarization converter for projection displays," Appl. Opt., vol. 36, pp. 6453-6457, 1997.

[4] H. Tanase, J. Mamiya, and M. Suzuki, "A new backlighting system using a polarizing light pipe," IBM J. Res. Dev., vol. 42, pp. 527-537, 1998.

[5] Z. Ge and S. T. Wu, "Nanowire grid polarizer for energy efficient and wide-view liquid crystal displays," Appl. Phys. Lett., vol. 93, p. 121104 , 2008.

[6] G. P. Bryan-Brown and J. R. Sambles, "Polarization conversion through the excitation of surface plasmons on a metallic grating," $J$. Mod. Opt., vol. 37, pp. 1227-1232, 1990.

[7] I. R. Hooper and J. R. Sambles, "Surface plasmon polaritons on narrowridged short-pitch metal gratings in the conical mount," J. Opt. Soc. Am. $A$, vol. 20, pp. 836-843, 2003. 
[8] I. R. Hooper and J. R. Sambles, "Broadband polarization-converting mirror for the visible region of the spectrum," Opt. Lett., vol. 27, pp. 2152-2154, 2002.

[9] N. Passilly, K. Ventola, P. Karvinen, P. Laakkonen, J. Turunen, and J. Tervo, "Polarization conversion in conical diffraction by metallic and dielectric subwavelength gratings," Appl. Opt., vol. 46, pp. 4258-4265, 2007.

[10] C. C. Tsai and S. T. Wu, "Broadband wide-angle polarization converter for LCD backlight," Appl. Opt., vol. 47, pp. 2882-2887, 2008.

[11] C. C. Tsai and S. T. Wu, "Study of broadband polarization conversion with metallic surface-relief gratings by rigorous coupled-wave analysis," J. Opt. Soc. Amer. A, vol. 25, pp. 1339-1348, 2008.

[12] J. M. Jonza, M. F. Weber, A. J. Ouderkirk, and C. A. Stover, "Polarizing Beam-Splitting Optical Component," U.S. Patent 5962 114, Oct. 5, 1999.

[13] R. A. Depine and C. I. Valencia, "Reciprocity relations for s-p polarization conversion," Opt. Commun., vol. 117, pp. 223-227, 1995.

[14] A. V. Kats and I. S. Spevak, "Analytical theory of resonance diffraction and transformation of light polarization," Phys. Rev. B, vol. 65, p. 195406, 2002.

[15] M. G. Moharam, D. A. Pommet, E. B. Grann, and T. K. Gaylord, "Stable implementation of the rigorous coupled-wave analysis for surface-relief gratings: Enhanced transmittance matrix approach," J. Opt. Soc. Amer. A, vol. 12, pp. 1077-1086, 1995.

[16] P. C. Logofatu, S. A. Coulombe, B. K. Minhas, and J. R. McNeil, "Identity of the cross-reflection coefficients for symmetric surface-relief gratings," J. Opt. Soc. Amer. A, vol. 16, pp. 1108-1114, 1999.

[17] E. D. Palik, Handbook of Optical Constants. San Diego, CA: Academic, 1997.
Chang-Ching Tsai received the Ph.D. degree in optics from College of Optics and Photonics, University of Central Florida, Orlando.

His current research interests include liquid crystal displays, laser beam steering, and sub-wavelength optics.

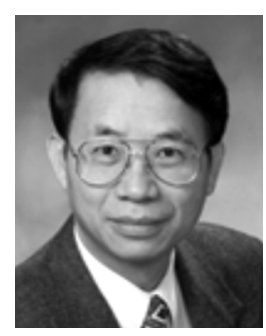

Shin-Tson Wu (M'98-SM'99-F'04) received the B.S. degree in physics from National Taiwan University, and the Ph.D. degree from the University of Southern California, Los Angeles.

$\mathrm{He}$ is a PREP professor at College of Optics and Photonics, University of Central Florida (UCF). His studies at UCF concentrate in liquid crystal displays, liquid crystal materials, optical communications, photonic crystal fibers, and bio-photonics. Prior to joining UCF in 2001, he worked at Hughes Research Laboratories, Malibu, CA, for 18 years. He has co-authored 5 books: Introduction to Flat Panel Displays (Wiley, 2008, with J. H. Lee and D. N. Liu), Fundamentals of Liquid Crystal Devices (Wiley, 2006, with D. K. Yang); Introduction to Microdisplays (Wiley, 2006, with D. Armitage and I. Underwood), Reflective Liquid Crystal Displays (Wiley, 2001, with D. K. Yang) and Optics and Nonlinear Optics of Liquid Crystals (World Scientific, 1993, with I. C. Khoo), 6 book chapters, over 300 journal publications, and more than 55 issued patents.

Dr. Wu is a recipient of SPIE G. G. Stokes award and SID Jan Rajchman prize. He is a Fellow of the Society of Information Display (SID), Optical Society of America (OSA), and SPIE. 